\title{
ERRATUM
}

\section{Penetration of Candida Biofilms by Antifungal Agents}

Mohammed A. Al-Fattani and L. Julia Douglas

Division of Infection and Immunity, Institute of Biomedical and Life Sciences, University of Glasgow, Glasgow, United Kingdom

Volume 48, no. 9, p. 3291-3297, 2004. Page 3291: The first sentence of the abstract should read "A filter disk assay was used to investigate the penetration of antifungal agents through single- and mixed-species biofilms containing Candida." 